\title{
Artificial Neural Network Model for Cutoff Frequency Calculation for Denoising of ECG Signal Using FIR Filter
}

\author{
Gaurav jain ${ }^{1}$ and R. P. Narwaria ${ }^{2}$ \\ Department of Electronics Engineering Madhav Institute Technology and Science \\ Gwalior, India
}

\begin{abstract}
In this paper, an automated approach in electrocardiogram (ECG) Signal, by noise reduction using artificial neural network is introduce. First, 30 of noise heart signal are collect from MIT-BIH database system. cutoff frequency is calculated by transforming to frequency domain. Since heart signals are at low pass frequency, a Finite Impulse Response (FIR) filter is enough to remove the noise. In the next stage, a dataset is configures for a multilayer perceptron (MLP) trained with feedforward algorithm. The approximate value of SNR gives the information about the reduction of noise.
\end{abstract}

Keywords: Finite Impulse Response (FIR),Low-pass filter, Cutoff frequency, ECG data signal, Multilayer Percecptron(MLP),Power Spectral Density(PSD),Signal to Noise Ratio(SNR)

\section{Introduction}

The electrocardiogram (ECG) is a process that is used to information about the electrical and muscular activity of the heart. It is a relative simple test to perform, the reading of the ECG tracing requires significant amounts of training.Electrical activity of heart can be displayed by electrocardiogram signal (ECG)[3]. The implementation of FIR filter having based on three terms: the cutoff frequency $\omega \mathrm{c}$, the filter $\operatorname{order}(\mathrm{N})$ and the window size.The order of filter determine the width of transition.Higher order of filter gives the sharp cutoff in frequency response[1]. FIR channels were chosen over IIR as they are steady and the impact of limited word length on the cutoff frequency or time duration is smaller than that for Infinite Impulse Response (IIR) channels[2]. Power Spectral Density (PSD) is a measure of intensity of signal power, In the frequency domain. the PSD is computed from the FFT spectrum of a signal.Signal-to-ratio(SNR) is defined as the dimensionless ratio of signal power to noise power.SNR is usually in decibel $(\mathrm{dB})$. The removal of noise in ECG signal by improving the SNR ratio, it affect the ECG signal to reduce noise. Figure1, shows the ECG signal is a typical pulse comprises of a three sections: $\mathrm{P}$ wave, QRS complex and $\mathrm{T}$ wave. The $\mathrm{P}$-wave represents depolarization of the atria, the QRS complex represents the rapid depolarization of the right and left ventricles and the T-wave represents the repolarization of the ventricles. 


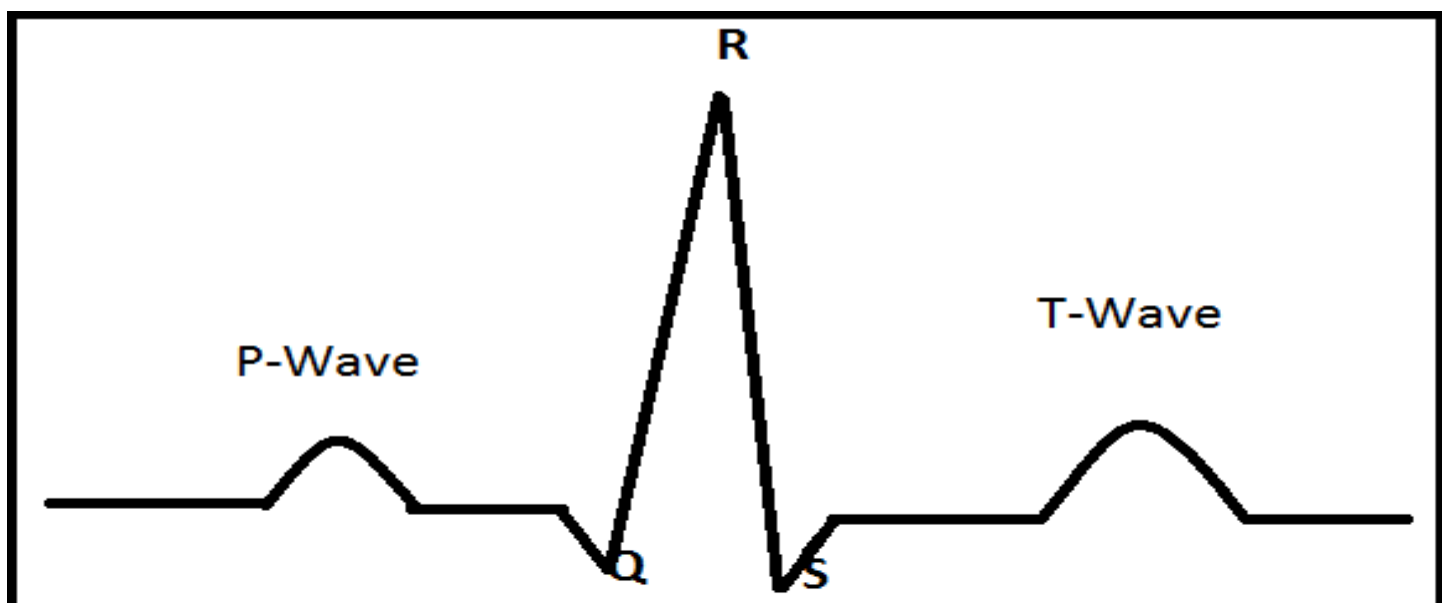

ECG signal of normal heart

Figure 1. ECG Signal from a Normal Human

In this paper the ECG signal is vulnerable to noise artifact and it is require to noise removing using filter in artificial neural network [7]. First 30 of noisy heart signal are collected from MIT-BIH data base. In the spectrum of the ECG signal is extracted from the three classes: arrhythmia, supraventricular and normal ECG signal [5]. By using Fast Fourier Transform(FFT) the ECG signal convert from time signal to frequency signal.[6] To reducing the noise from ecg signal,we calculate the parameters having the noise varied i.e. Mean,Variance,Standard deviation,Power Spectral density[8].

\section{Methodology}

\subsection{Database}

Physiobank is a collection data of heart signal of ordinary and patient human. In this research, 30 of ECG samples are collect from three databases[8]. MIT-BIH Arrhythmia Database that is collection of 48 completely annotated on half-hour two-lead ECG, MIT-BIH Normal sinus Rhythm Database that integrates 18 long haul ECG recordings of subjects referred to the Arrhythmia Lab. at Boston Beth Israil Hospital and MIT-BIH Supraventricular Arrhythmia Database that integrates 78 half-hour ECG recordings supplemented the case of supraventricular arrhythmias in the MIT-BIH Arrhythmia Database [4]. To remove the noise disruption of ECG signal FIR is effective to normalized Cutoff frequency. The objective behind this is to calculate the cutoff frequency by neural network system.Consider the noise interference in ECG signals, All 30 sample Signals must be transforms to frequency domain by using Fast Fourier Transform (FFT).Figure show examples of noisy signals and Fourier transform. Figure 2, shows the sample of ECG signal and noisy signal after the baseline remove from original signal. 


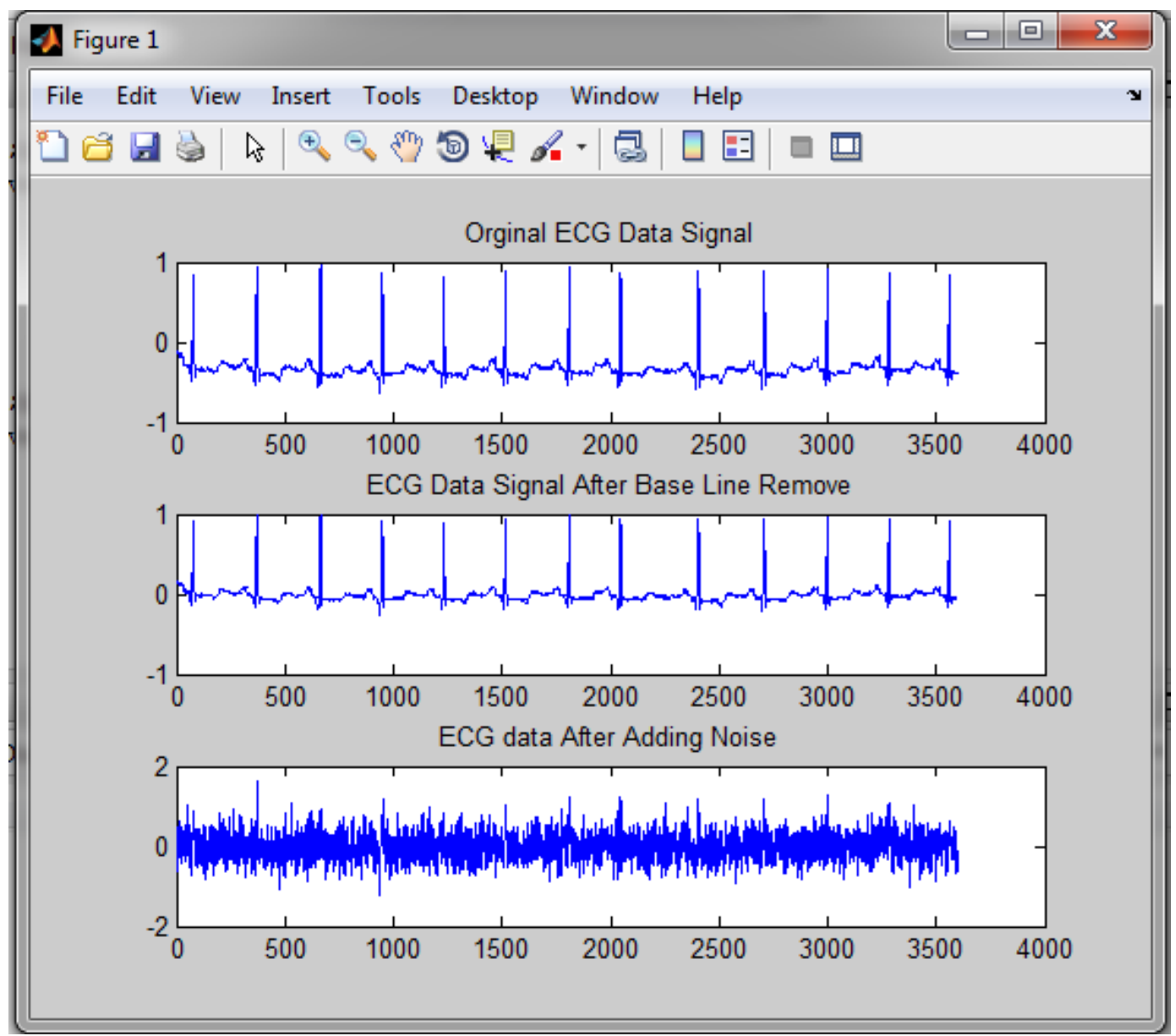

Figure2. ECG Signal with Noise

\subsection{Dataset Configuration}

To configure the dataset by transforming signal to frequency domain are extracted. Standard deviation in view of definition is as shown:

$$
\sigma=\sum_{n=0}^{N-1} \frac{1}{N}\left(x_{n}-\mu\right)^{2}
$$

Where $\mathrm{N}$ is number of tests, $\mathrm{Xn}$ is information test and $\mu$ is the mean. $\sigma$ is the standard deviation [4].

Utilizing Matlab programming, feature value are removed from Fourier transform of each signal and resulting value are collected in a dataset. Table 1, is collection of dataset. Since the previous method depends on supervised learning for neural system, therefore it is important to compute value for every record of information in a dataset. The objective is the estimation of FIR Cutoff Frequency Calculating for ECG Signal Noise Removing Using ANN cutoff frequency that can be computed utilizing frequency range. At that point the frequency must be standardized. Where Fs is the sample frequency. 
Table 1. Dataset of Calculated Value

\begin{tabular}{|c|c|c|c|c|c|c|c|}
\hline $\begin{array}{l}\text { S.N } \\
0\end{array}$ & Signal & $\begin{array}{l}\text { Mean } \\
\text { FFT }\end{array}$ & $\begin{array}{l}\text { Variance } \\
\text { FFT }\end{array}$ & $\begin{array}{l}\text { Standard } \\
\text { Deviation }\end{array}$ & PSD & $\mathrm{SNR}(\mathrm{db})$ & $\omega c$ \\
\hline 1 & Super ventricular & .133 & 20.83 & 4.56 & .122 & -9.01 & .20 \\
\hline 2 & Super ventricular & .049 & 32.785 & 5.72 & .175 & -7.8 & .33 \\
\hline 3 & Super ventricular & .1367 & 63.7 & 7.23 & .225 & -11.4 & .266 \\
\hline 4 & Super ventricular & .1455 & 52.36 & 7.91 & .245 & -13.6 & .40 \\
\hline 5 & Super ventricular & .1347 & 59.6 & 6.95 & .216 & -10.7 & .36 \\
\hline 6 & Super ventricular & .178 & 62.39 & 7.94 & .303 & -11.4 & .22 \\
\hline 7 & Super ventricular & .044 & 22.78 & 5.97 & .278 & -12.8 & .28 \\
\hline 8 & Super ventricular & .591 & 44.86 & 5.67 & .237 & -13.7 & .40 \\
\hline 9 & Super ventricular & .076 & 56.56 & 4.87 & .314 & -11.9 & .66 \\
\hline 10 & Super ventricular & .049 & 61.56 & 7.56 & .231 & -13.7 & .44 \\
\hline 11 & Normal ECG & .054 & 18.56 & 4.39 & .186 & -5.7 & .33 \\
\hline 12 & Normal ECG & .140 & 52.36 & 7.23 & .315 & -17.54 & .28 \\
\hline 13 & Normal ECG & .025 & 218.94 & 14.8 & .221 & -23.45 & .44 \\
\hline 14 & Normal ECG & .276 & 372.56 & 18.56 & .287 & -25.77 & .80 \\
\hline 15 & Normal ECG & .017 & 40.87 & 6.44 & .198 & -13.58 & .60 \\
\hline 16 & Normal ECG & .049 & 21.89 & 4.37 & .212 & -17.77 & .22 \\
\hline 17 & Normal ECG & .041 & 18.45 & 3.98 & .201 & -11.47 & .20 \\
\hline 18 & Normal ECG & .386 & 23.56 & 4.77 & .203 & -16.49 & .33 \\
\hline 19 & Normal ECG & .457 & 21.56 & 4.56 & .235 & -9.54 & .26 \\
\hline 20 & Normal ECG & .043 & 15.22 & 6.9 & .168 & -7.65 & .166 \\
\hline 21 & Arrhythmia & .096 & 221.23 & 14.56 & .153 & -26.45 & .66 \\
\hline 22 & Arrhythmia & .127 & 216.45 & 13.54 & .143 & -24.45 & .66 \\
\hline 23 & Arrhythmia & .261 & 126.35 & 11.56 & .189 & -19.74 & .44 \\
\hline 24 & Arrhythmia & .211 & 70.89 & 9.82 & .237 & -17.4 & .40 \\
\hline 25 & Arrhythmia & .295 & 139.46 & 11.56 & .269 & -21.67 & .60 \\
\hline 26 & Arrhythmia & .18286 & 115.46 & 13.45 & .287 & -19.68 & .44 \\
\hline 27 & Arrhythmia & .114 & 201.45 & 13.74 & .234 & -24.89 & .66 \\
\hline 28 & Arrhythmia & .367 & 175.23 & 12.56 & .246 & -20.12 & .80 \\
\hline 29 & Arrhythmia & .187 & 164.56 & 9.56 & .257 & -18.86 & .76 \\
\hline 30 & Arrhythmia & .156 & 159.56 & 13.56 & .219 & -17.77 & .64 \\
\hline
\end{tabular}

\subsection{MLP Training}

A multilayer perceptron (MLP) is mapping of sets of input data onto a set of appropriate outputs by using a feedforward artificial neural network model. An MLP consists of multiple layers of nodes, with each layer fully connected to the next one [11].Multilayer Perceptron (MLP) is a proficient neural system for grouping issues furthermore, it has an extensive execution. There are basic terms required in preparing the MLP system:

- Number of hidden node

- Number of layers in hidden node

- Converging to an ideal arrangement in a sensible timeframe

- Testing neural system for overfitting

The MLP with 3 layers in input node and 1 layer in output node is prepared. 30 numbers of records of dataset will prepare the neural system and 10 records are utilized to test. Variable of different tests to get the gain of significant performance.Figure4 shows the MLP for neural network. 


\section{MSE Performance}

Mean Square Error (MSE) gives the best optimize value of cutoff frequency at which the filter denoising the ECG signal.From the neural network,the best performance validation at .052805 , At this value of cutoff frequency the neural network is to more efficient to denoising the ECG signal.Figure4 shows the MSE performance of ECG signal. Table 2, contains the optimize SNR correspond to their cutoff frequency.

Table 2. Optimization of SNR with Cutoff Frequency

\begin{tabular}{|l|l|}
\hline $\begin{array}{l}\text { Optimize value of } \\
\text { SNR }\end{array}$ & $\begin{array}{l}\text { Optimize cutoff } \\
\text { frequency }\end{array}$ \\
\hline-7.1201 & 0.77016 \\
\hline-7.5751 & 0.41078 \\
\hline-8.2293 & 0.48486 \\
\hline-7.1462 & 0.41256 \\
\hline-7.9263 & 0.46512 \\
\hline-10.1996 & 0.47738 \\
\hline-10.8512 & 0.41241 \\
\hline-11.9728 & 0.54964 \\
\hline-11.1789 & 0.36729 \\
\hline-7.0522 & 0.50729 \\
\hline
\end{tabular}

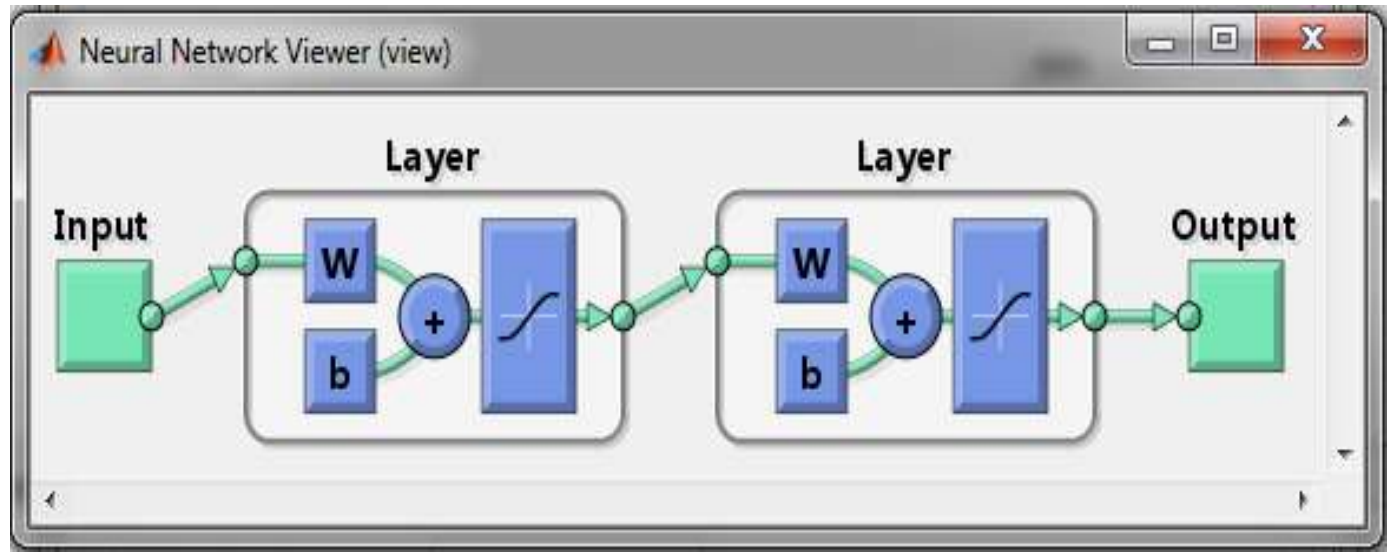

Figure 4. Neural Network Multilayer Perceptron for Input and Output Layer 


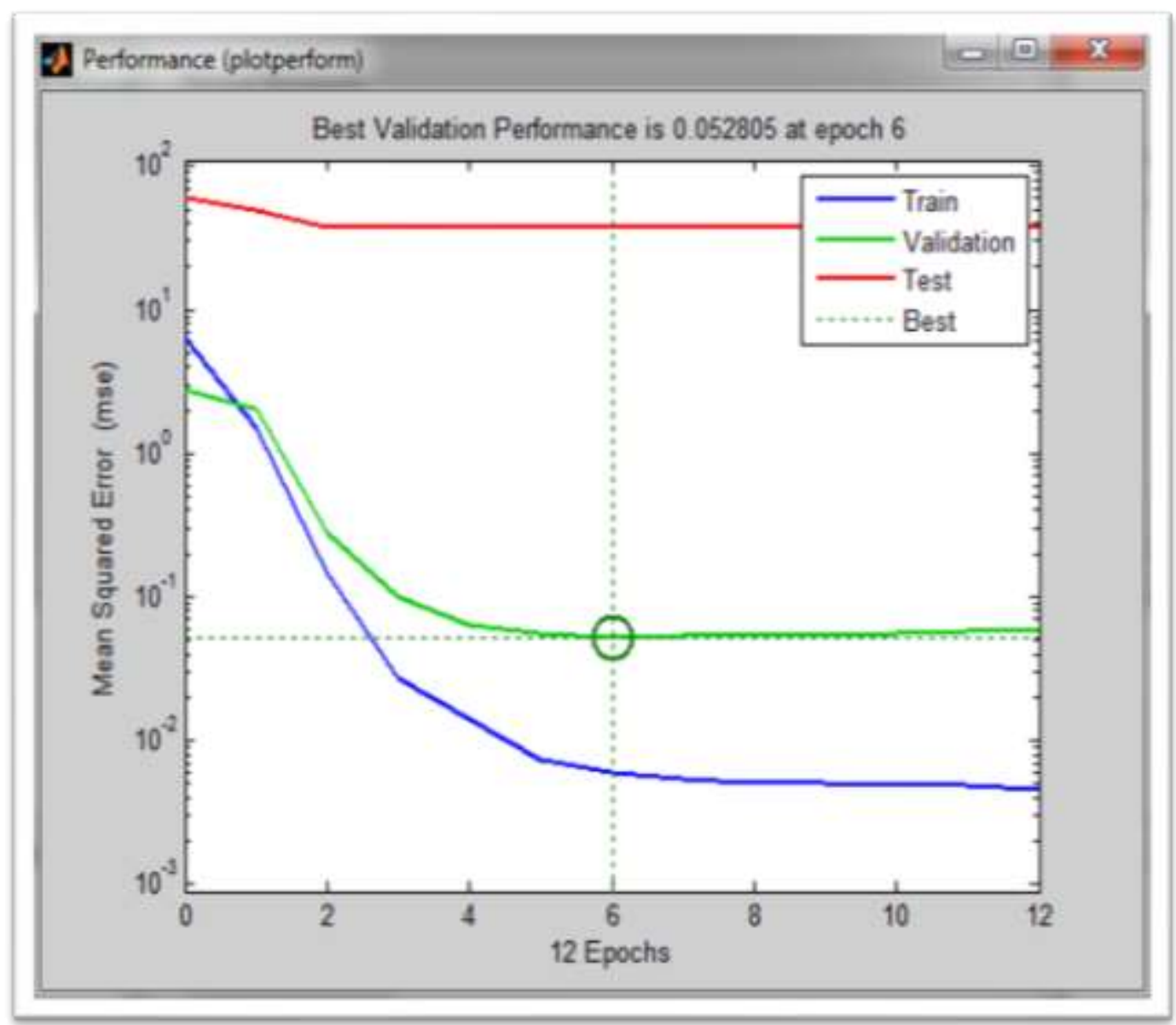

Figure 5. MSE Performance of ECG Signal After Denoising

\section{Conclusion}

Results demonstrate that neural system can be connected for taking care of issues of signal handling. The average power and signal to noise ratio was expressed out to compare the effecof noise on ECG signal. It has important execute for cutoff frequency and observing figures gives information about variation and tables demonstrates that the valuation of system is to effective. It demonstrates that the result is more than $90 \%$ to reduce.. For future works, we can also use to different type of window technique is used to denoise the ECG signal.

\section{References}

[1] K.D. Chinchkhede "On the implementation of FIR Filter with various window for enhancement of ECG signal”, IJEST, vol. 3, no. 3, (2011) March.

[2] M. Chavan, "FIR Equiripple digital filter for reduction of power line interference in the ECG Signal",ISPRA, (2008).

[3] M. K. Islam, "Study and Analysis of ECG Signal Using MATLAB \&LABVIEW as Effective Tools" IJCEE, vol. 4, no. 3, (2012) June.

[4] S. Moein, "FIR Cutoff Frequency Calculating for ECG Signal Noise Removing Using Artificial Neural Network" Verlag Berlin Heidelberg, ( 2010), pp.124-131.

[5] M. D. Choudhary and R. P. Narwaria " suppression of noise in ECG signal low pass IIR filters" IJECSE,vol.1, no. 4, (2012), pp 2238-2243

[6] M. Ferdjallah and R. E. Barr, "Frequency-domain digital filtering techniques for the removal of powerline noise withapplication to the electrocardiogram”, Comput Biomed Res.; vol. 23, no. 5, (1990) October, pp. 473-89. 
[7] S. Verma "Reduction of Artifact by FIR Low Pass Filter usingTriangular, Rectangular and Parzen windows" published in International Journal of Computer Information systems, vol. 5, no. 5, (2012), pp 75-85

[8] S. Bittoliya and R. P. Narwaria, "Intelligent ECG signal noise removal with moving median filter using neural network" IMPACT: IJRET ISSN 2321-8843, vol. 1, no. 3, (2013) August, pp. 77-84

[9] N. Aggarwal S. Thapar and P. Kaur "Design a low pass FIR low pass filter using particle swarm optimization Based Artificial Neural network", IJETTCS, vol. 1, no. 4, (2012).

[10] L. Badri, "Development of Neural Networks for Noise Reduction", The International Arab Journal of Information Technology, vol. 7, no. 3, (2010) July.

[11] S Moein, "Hepatitis Diagnosis by Training A MLP Artificial Neural Network" In: Worldcomp2008 Conference, Las Vegas, USA (2008).

[12] S. T. Moein, "Advances in Computational Biology: A MLP Neural Network for ECG NoiseRemoval Based on Kalman Filter", In: Arabnia, H.R. (ed.). Springer, Heidelberg, (2010).

[13] R. D. Wagh, "Noise Removal from Electrocardiogram (ECG) a Comparison Approaches" IJARCET) vol. 3, no. 1, (2014) January.

[14] S. Kasar, "Performance of Digital filters for noise removalfrom ECG signals in Time domain" IJIREEICE, vol. 2, no. 4, (2014) April.

[15] S. Moein, "Intelligent ECG Signal Noise Removal using PSONN" IJCA(0975 - 8887), vol. 45 no. 6, (2012) May.

[16] W. X. Zheng, "A Least- Squares based algorithm for FIR filtering with noisy data", IEEE (2003).

[17] M. Kaur and J. S. Ubhi, "Digital Filteration of ECG Signal for Removal of Baseline Drift", IACSIT (2011).

[18] Archive of ECG signals, https://www.physionet.org/cgi-bin/atm/ATM "MIT-BIH Arrhythmia Database". 
International Journal of Signal Processing, Image Processing and Pattern Recognition Vol. 10, No. 1 (2017) 